\title{
A Novel Approach to Estimate Diffuse Attenuation Coefficients for QuickBird Satellite Images: A Case Study at Kish Island, the Persian Gulf.
}

\begin{abstract}
Diffuse attenuation coefficient $(\mathrm{k} \mathrm{d})$ is a critical parameter for benthic habitat mapping using remotely sensed data. This research attempted to develop a new approach to estimate $\mathrm{k} \mathrm{d}$ in blue and green bands of QuickBird satellite image based on the integration of Lyzenga's method and updated NASA-k d 490 algorithm. To do this, the Lyzenga's method was utilized to determine the ratio of $\mathrm{k} \mathrm{d}$ in different bands of QuickBird satellite image. Additionally, NASA-k d 490 algorithm was applied to determine k d 490 by using remotely sensed reflectance values of blue ( $\mathrm{R}$ rs Blue ) and green ( $\mathrm{R}$ rs Green ) bands in each pixel of QuickBird satellite image. Since the aforementioned algorithm has been developed for other types of sensors, an approach using weighted mean value of parameters for SeaWiFS, MERIS, VIIRS, and OCTS sensors were employed to estimate parameter values for QuickBird image. After determining the $\mathrm{k} \mathrm{d} 490$ values as $\mathrm{k} \mathrm{d}$ for blue band, the $\mathrm{k} \mathrm{d}$ values for green and red bands were subsequently obtained by using Lyzenga's method. Then, Mumby and Edwards' method was employed as evidence to evaluate the accuracy of the results achieved from newly developed approach. Eventually, the maximum likelihood classifier was implemented during pre and post correction steps to examine the capability of the proposed approach. The final results proved to be consistent in the areas deeper than $2 \mathrm{~m}$ between estimated $\mathrm{k} \mathrm{d}$ values using the proposed approach and the results obtained from Mumby and Edwards' method. On the other hand, the values estimated for extremely shallow areas seem to be overestimated. Furthermore, results demonstrated an increment of $\sim 16 \%$ in the overall accuracy of the classification.
\end{abstract}

Keyword: Diffuse attenuation coefficient, Remote sensing, QuickBird, Benthic habitats, NASA-kd 490 